\title{
A Case Study on Master Students with the Textile Background in Tackling Fiber Identification Problems
}

\author{
Yue-qi ZHONG ${ }^{1,2,{ }^{*}}$ \\ ${ }^{1}$ College of Textiles, Donghua University, Shanghai, China, 201620 \\ ${ }^{2}$ Key Laboratory of Textile Science \& Technology, Ministry of Education, Donghua University, \\ Shanghai 201620, China \\ ${ }^{*}$ Corresponding author
}

Keywords: Wool, Cashmere, SEM, SURF, SVM, Deep learning.

\begin{abstract}
The identification of Wool/Cashmere fibers is an extremely challengeable problem since both fibers possess highly similar morphological features. In this research, we monitored two group of master students with the textile background in tackling this specific task. Both groups have no experience on machine vision. Two learning curves, machine learning, and deep learning were randomly distributed to the groups for them to learn. The entire experimental procedure was carried under the same condition, i.e., computing resources, laboratory facilities, working environment, and weekly discussion with an academic advisor. From our observation, higher accuracy has been reached by the group following the path of deep learning. Comparatively, knowledge of image analysis or hand-crafted feature extraction has empowered the students following the path of machine learning with a more fundamental understanding of feature extraction.
\end{abstract}

\section{Introduction}

In textile/apparel industry, both wool (sheep hair) and cashmere fibers (goat hair) are highly popular raw materials for various end products. However, cashmere is considered as one of the "luxury fiber". It is not uncommon that underground interest parties profit from adulterating cashmere with fine wool. Hence the task of distinguishing cashmere from wool is a key challenge to protect consumer benefits and to maintain the justice of the market.

Technically, the identification methods on wool/cashmere can be divided into light microscopy, scanning electron microscopy, and DNA methods. Skinkle [1] proposed that fiber diameter and height of scales can be adopted to distinguish wool from cashmere. Zhong et al [2] transferred the microscopic images into projection curves. Numerical features were extracted accordingly and were fed into different classifiers. Lu et al [3] proposed the bag-of-words with spatial pyramid match and made great progress. On the scanning electron microscopy, Ryder and Wortmann $[4,5]$ proposed a multi-parameters method which greatly improved the speed of fiber recognition. Different from these morphological approaches, DNA method [6] achieves high accuracy by extracting mitochondrial DNA from fibers followed by PCR for amplification and sequencing. The cashmere and wool fiber can be identified by the specific base sequences. However, it is an expensive and time-consuming approach. Actually, the procedure of wool/cashmere identification is a typical problem of feature extraction, which is the input of classification. In this sense, handcrafted features were proposed such as SIFT [7], SURF [8] followed by machine learning algorithms such as Bayesian method or SVM classifier.

As an effort to tackle the problem of wool/cashmere identification, and to train master students towards a successful career path, we try to compare the output of academic training on two group of students with different learning curves/paths. To simplify the problem, we use scanning electron microscopy (SEM) images as the input for fiber classification since the quality of the image is more promise. 


\section{Methodology}

\section{Profile of Groups}

Two group of male master students with the similar grade and background were selected as the subjects in this study. Both have no previous knowledge of computer science. During the entire training procedure, they were exposed to the same learning condition with the same computing resources, laboratory facilities, and working environment. Both groups had to discuss with the same academic advisor on a weekly basis.

\section{Learning Curve}

Two different learning curves were designed for the groups to learn. For the convenience of explanation, we denote them as $\mathrm{C} 1$ and $\mathrm{C} 2$ respectively. The core knowledge module of each curve was demonstrated in Table 1.

Table 1. Core knowledge modules designed for $\mathrm{C} 1$ and $\mathrm{C} 2$.

\begin{tabular}{|l|l|l|l|}
\hline Learning curve & Grade 1 module (basic) & Grade 2 module (medium) & Grade 3 module (advanced) \\
\hline C1 & Image processing & Machine learning & Feature extraction \\
\hline C2 & Neural network & Deep learning & Network architecture design \\
\hline
\end{tabular}

From our understanding, $\mathrm{C} 1$ is prone to the traditional machine learning method, while $\mathrm{C} 2$ is more deep-learning likely. $\mathrm{C} 1$ will fabricate the subjects with more knowledge on how to hand-craft the embedded feature. And C2 will deliver the state-of-the-art technology on artificial intelligence.

\section{Experimental Design $(\mathrm{C} 1)$}

The same raw material of wool and cashmere were prepared for SEM photography. Both groups have the same pre-labeled data set. For group members follow the learning curve of $\mathrm{C} 1$, the detailed procedure follows (Fig. 1).

\section{A. Image background removal}

Step 1. Use Sobel filter for the edge detection.

Step 2. Detect the intersection of the skeleton area and the edge of the image, and then expand the fiber edge to get a closed fiber area.

Step 3. Use the mathematical morphology to fill the holes in the fiber. Fiber area parameter is used to remove remaining noised region to get the segmented image.

Step 4. Mask the segmented image to remove the background from the original image.

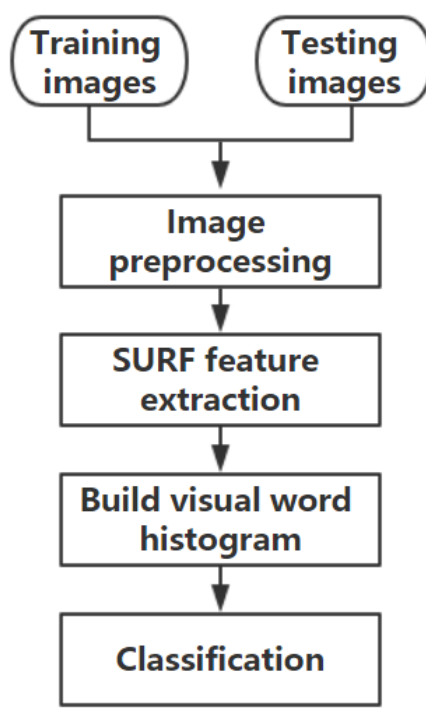

Figure 1. Technical pipeline for group 1 with $\mathrm{C} 1$ 


\section{B. SURF feature extraction and bag-of-words}

SURF algorithm proposes a solution which can find the key points inside a certain type of image such as corner points, edge points, highlight points and then distinguish them between different types. It uses the integral image to speed up the feature acquisition. Given an extracted SURF feature set, k-means clustering can generate $\mathrm{k}$ cluster center randomly from the dataset. After minimizing the Euclidean distance of each sample point to each center, the central point of each cluster can be regarded as the visual word that describes the characteristics of the fibers. In this way, the input image is transformed into a visual histogram containing the key information of the fiber, which can be employed as the input for classification. In group \#1's approach, Support vector machine (SVM) has been adopted as the final classifier.

\section{Experimental Design (C2)}

For the group following the learning curve of $\mathrm{C} 2$, a deep learning approach was performed. Due to the small amount of SEM image, data augmentation was carried (Fig. 2).

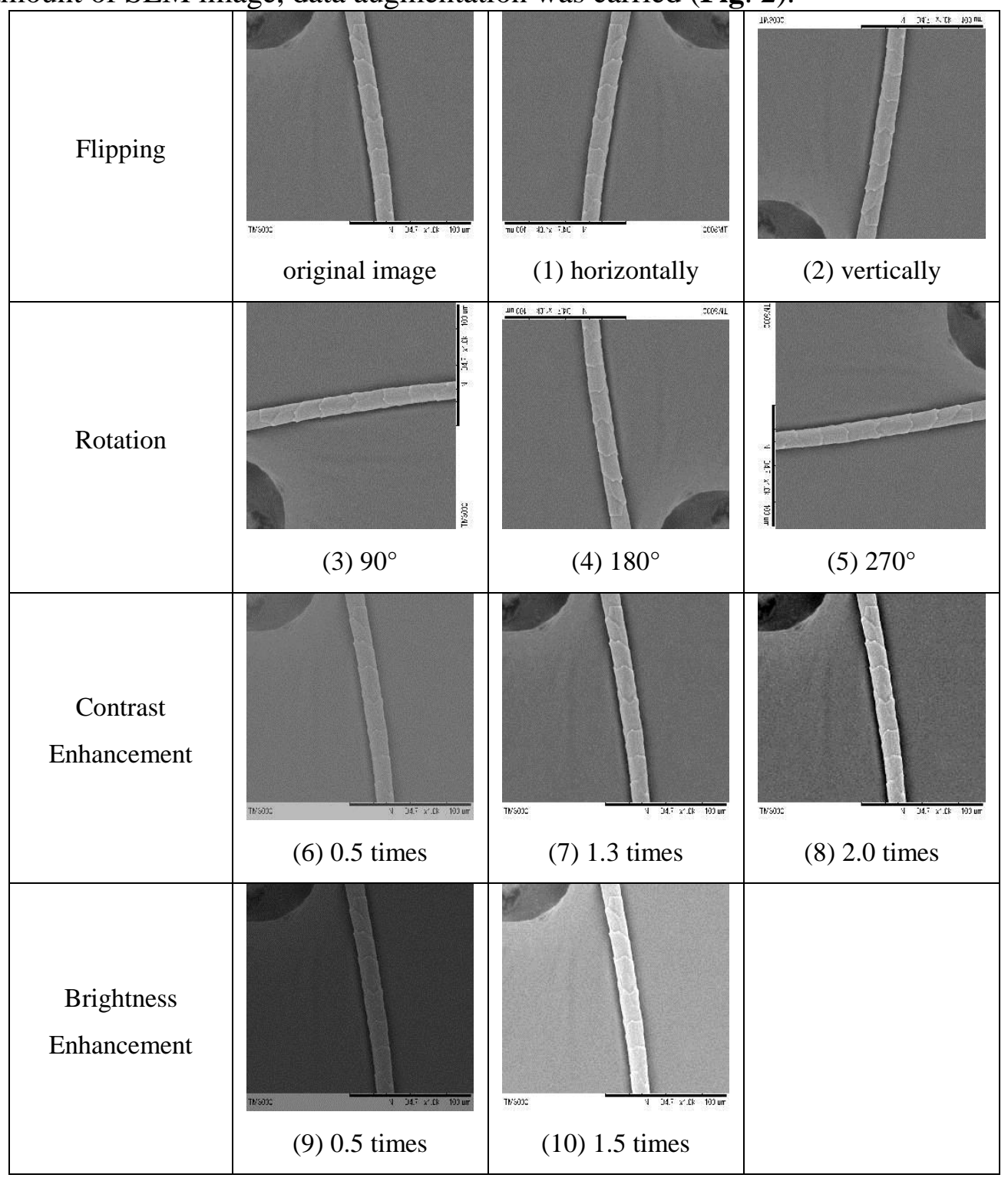

Figure 2. Operations for data augmentation

Residual Network (ResNet) [9, 11] was employed as the deep learning architecture. ResNet featured special shortcut connections and a heavy use of batch normalization (BN) [10]. ResNet is practically easier to train compared with other architecture with the same parameters. In this work, group \#2 chose ResNet-50 (50 layers) to train the datasets. 


\section{Results and Discussion}

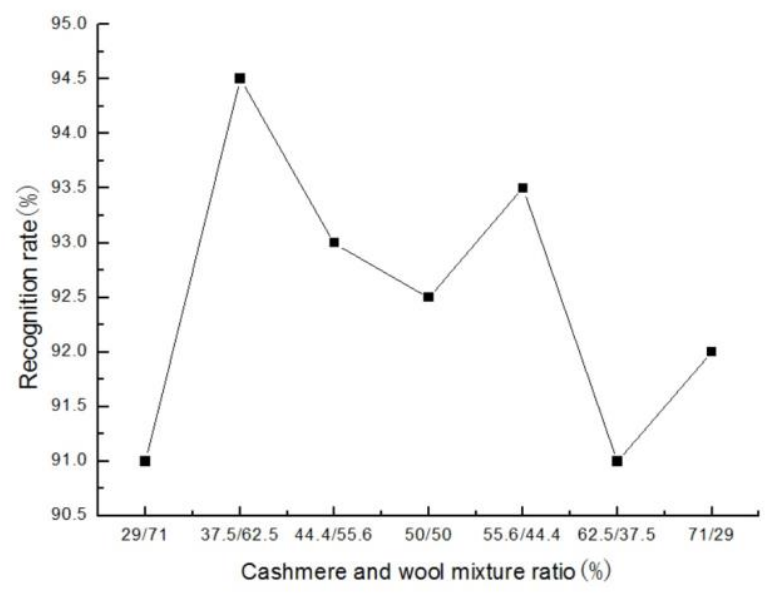

Figure 3. Cashmere/wool identification results based on different mixture ratio.

Both groups test their solution on a PC with Intel Core i7-6500U CPU at 2.50G Hz, and $8 \mathrm{G}$ memory plus $8 \mathrm{G}$ GPU memory for acceleration. Fig. 3 shows the identification results of two fibers in different mixing ratios based on images with background removal. The lowest average accuracy is $91 \%$, and the CV of the classification under various blends is 0.014 , which means the performance of this method is very stable. The average training time is 120 seconds, and the testing time is 16 seconds.

Table 2. Accuracy and recall of group 2's deep learning approach

\begin{tabular}{|c|c|c|c|}
\hline Reality Prediction & Cashmere & Wool & Recall \\
\hline Cashmere & 749 & 7 & $99.07 \%\left(\mathrm{R}_{\mathrm{c}}\right)$ \\
\hline Wool & 12 & 718 & $98.36 \%\left(\mathrm{R}_{\mathrm{w}}\right)$ \\
\hline Accuracy & $98.42 \%\left(\mathrm{~A}_{\mathrm{c}}\right)$ & $99.03 \%\left(\mathrm{~A}_{\mathrm{w}}\right)$ & \\
\hline
\end{tabular}

Table 2 demonstrates the result of group \#2 with deep learning as the final solution. Obviously, data augmentation works as its expectation in dealing with the small dataset. The average training time is 2.3 hours, and the testing time is less than 2 seconds.

After interviewing both groups, we find that students in group \#1 have learned more fundamental knowledge of image segmentation, SURF, SIFT feature extraction, and bag-of-words in computer vision. They feel more confident in using handcrafted features for image recognition and/or classification problems. As for group \#2, students learned the entire pipeline of deep learning architecture including network selection and hyperparameter fine tuning. Both groups have a better understanding of either machine learning or deep learning. However, due to the fact that the final accuracy reported by group \#1 is lower than that of group \#2, it implies hand-crafted algorithms might not be the best solution for wool/cashmere identification. Furthermore, the efficiency of group \#1 is lower than that of the group \#2, which indicate the traditional pipeline should be re-evaluated towards the real world challenges.

\section{Summary}

In this paper, we observed and studied the output of two groups of master students with a textile background in tackling a computer vision problem under the same condition. It is found that deep learning is easier to be learned and the output is better than that of the machine learning. This could be 
a reasonable cause in re-design the curriculum and the educational method for students majored in textile science and technology.

\section{Acknowledgement}

This research was financially supported by the National Natural Science Foundation of China (Grant No. 61572124).

\section{References}

[1] Skinkle JH. The Determination of Wool and Mahair by Seale Size and Diameter. Amer Dyest: 1936, 25:620-621.

[2] Zhong YQ, Lu K, Tian J, Zhu H. Wool/cashmere identification based on projection curves. Textile Research Journal: 2016, 87(14): 1730-1741.

[3] Lu K, Zhong YQ, LI D, Chai XY, Xie HY, Yu ZC, Tayyab N. Cashmere/wool identification based on bag-of-words and spatial pyramid match. Textile Research Journal: 2017 DOI: 10.1177/0040517517723027.

[4] Ryder ML. Goat Fibre and Its Production. Proceeding of Eighth International Wool Textile Research Conference, Christchurch, 1990, 2:241-266.

[5] Wortmann FJ. Light Microscopy of Yak and Cashmere Fibre Blends-State of the Art. Processing of Second International Symposium on Specialty Animal Fibers. Aachen, 1990. p. 113-120.

[6] Tang M, Zhang W, Zhou H, Fei J, Yang J, Lu WM, Zhang SY, Ye SH, Wang XP. A real-time PCR method for quantifying mixed cashmere and wool based on hair mitochondrial DNA. Textile Research Journal: 2014, 84(15): 1612-1621.

[7] Lowe DG. Distinctive Image Features from Scale-Invariant Keypoints. International Journal of Computer Vision: 2004, 60(2):91-110.

[8] Bay H, Ess A, Tuytelaars T, Gool LV. Speeded-Up Robust Features (SURF). Computer Vision \& Image Understanding: 2008, 110(3):346-359.

[9] He KM, Zhang XY, Ren SQ, Sun J. Deep Residual Learning for Image Recognition. 2016 IEEE Conference on Computer Vision and Pattern Recognition (CVPR), Las Vegas, NV, United States, 2016. p. 770-778.

[10] Sergey I, Szegedy C. Batch Normalization: Accelerating Deep Network Training by Reducing Internal Covariate Shift. International Conference on Machine Learning: 2015:448-456.

[11] He KM, Zhang XY, Ren SQ, Sun J. Identity Mappings in Deep Residual Networks. European Conference on Computer Vision: 2016:630-645. 